\title{
A Formal Computational Semantics and Pragmatics of Speech Acts*
}

\author{
Eric WERNER
}

\author{
University of Hamburg \\ Department of Computer Science, Project WISBER, P.O. Box 302762 \\ Jungiusstrasse 6, 2000 Hamburg 36, West Germany \\ and \\ and \\ Bowdoin College, Brunswick, Maine 04011, U.S.A.
}

\begin{abstract}
*This work was in part supported by the German Ministry for Research and Technology, BMFT, and was part of the joint project WISBER. The WISBER partners include: Nixdorf Computer AG, SCS GmbH, Siemens AG, the University of Hamburg and the University of Saarbruecken. It was also in part supported by grants from Bowdoin College.
\end{abstract}

\begin{abstract}
This paper outlines a formal computational semantics and pragmatics of the major speech act types. A theory of force is given that allows us to give a semantically and pragmaticaly motivated taxonomy of speech acts. The relevance of the communication theory to complex distributed artificial intellince, DAI, systems is described.
\end{abstract}

\section{Introduction}

In a system that engages in a dialogue with a user about some domain, like the one we are builing in the WISBER project [Bergmann, Gerlach 87], the problem of describing the meaning of a speech act in terms of its effect on the user model and, more generally, on the system's knowledge base becomes central. The fundamental problem is that, until now, there has been no general formal theory of meaning for speech acts. Previous formal semantic theories such as Montague [74] and situation semantics [Barwise and Perry 83] were limited to assertions. Cohen and Perrault [79] give only a theory of how speech acts are planned, the semantics being implicit in the add and delete lists of the operators. Appelt [85] gives a semantics for assertions based on Moore [80] that is compatible with our approach. However, there is no explicit theory of communication. As Appelt himself states, what is lacking is an explicit theory of intention. Searle and Vanderveken [85] do little more than classify speech acts based on features that were seen as relevant to their differentiation. However, as we will argue below, those features are not motivated by any coherent theory of meaning. The crucial features that define force are left unanalyzed. None of the above theories give a formal semantics for nonassertive speech acts. As Searle also states, what is missing is formal theory of intentions.

In this paper we outline a formal semantics and pragmaties of speech acts based on an explicit formal theory of information and intention. A formal description of the notion of illocutionary force is given. We take a new look at Searle's a classification of speech acts. In the process, we develop a deeper understanding of the rather vague notion of force.
We start with Searle's classification of illocutionary acts. Searle and Vanderveken's definition of force is criticized. Next Habermas is criticized. Next, referential theories of meaning are looked at critically. Then we present our theory of the semantics and pragmatics of speech acts. We then use that theory to give a theory of force. We then describe the speech acts in Searle's classification in terms of the semantic and pragmatic effects they have in the communicative situation. Finally we show how the semantics and pragmatics of speech acts is related to the general communcative process in the social world and show that the classification of speech acts really reflects the fundamental way agents use language to coordinate and make possible their social actions.

\section{Searle's Classification of Illocutionary Acts}

What distinguishes a request from an assertion? One answer is that their force is different. But what is force? According to Searle, when humans communicate they are engaged in an activity. An utterance, according to Searle, can be broken down into two basic components, the illocutionary force $F$ and the propositional content $p$. The utterance is symbolized as $F(p)$. In order to classify the different types of force $F$, Searle and Vanderveken [85] attempt to reduce the force of a speech act to more primitive features. The force and the propositional content is then used to divide speech acts into six general classes.

In Searle's classification of illocutionary acts [Searle 75] he distinguishes six general classes based on four dimensions. The four dimensions were the illocutionary point (assertive - , directive $!$, commissive $C$, expressive $\mathrm{E}$, declarative $\mathrm{D}$, and representative declarative $\mathrm{D}_{\mathrm{r}}$ ), the direction of fit (word-to-world $\downarrow$, world-to-word $\uparrow$ ), the psychological state (believes $B$, wants $W$, intends I), and the propositional content $p$. The null symbol $\Phi$ was used to indicate that a given dimension had no instance. The following speech act classes were distinguished:

1. Assertives: $\vdash \downarrow B(p)$ This says that the assertive $\vdash$ (the illocutionary point) has a direction of fit of word-to-world $\downarrow$, the psychological state $B$ for the sincerity condition to hold must be that the speaker belives $B$ the propositional content expressed p. Example: The speaker states The door is open and belives that the door is open. 
2. Directives: ! $\uparrow W(H$ does A.) This states that the directive ! has a direction of fit of world-to-word $\uparrow$, the psycholslgical state must be that the speaker wants that the heerer $\mathbf{H}$ do the action $\mathrm{A}$. Example: The speaker gives the command Open the door and wants the door to be opened. Note, here, as in the declaratives, we need certain role presuppositions to hold.

3. Commissives: $C \uparrow I(S$ does $A)$ The commissive $\mathrm{C}$ has a direction of fit of world-to-word $\uparrow$, the psychololgical state must be that the speaker $S$ intends $I$. to do the action A. Example: 'The speaker says I will open the door and intends to do it.

4. Expressives: E $\phi(\mathrm{P})(\mathrm{S} / \mathrm{H}+$ Property) The expressive $F$ has no direction of fit $\phi$, the psychological state should correspond to the emotion/attitude expressed by the speaker about the property of the spearker $\mathbf{S}$ or hearer $\mathrm{A}$. Wxample: The speaker exclaims I like your coat and neans $\mathrm{i}$ \%.

5. Doclarations: $D \leftrightarrow \phi(p)$ 'The declaration $D$ has a self-realizing direction of fit $\leftrightarrow$ (since the state described by the propositional content $p$ is realized by the very act of saying it). There is no pyschological state necessary and, hence, no sincerity condition. However, the speaker must have a certain institutional role, e.g., In saying I resign or You're fired the speaker must have the role of employee or boss, respectively.

6. Representative Declaratives: $\mathrm{O}_{\mathfrak{r}} \quad \downarrow \leftrightarrow B(p)$ The representative declarative has a direction of fit of word-to-world $\downarrow$ ( since the speaker bases his declaration on the recognition of some fact $p$ ), and a self-realizing directions of fit $\leftrightarrow$ (like declarations). There is a psychological state in the sincerity condition that the speaker must believe $B$ the proposition expressed. Example: 'The umpire declaring, You're out or the judge, $I$ find you guilty as charged.

\section{Critique of Searle's Notion of Force}

Note that the actual classes of illocutionary acts that Searle distinguishes correspond exactly to the values of the illocutionary point dimension. It turns out that there is a one to one correspondence between the illocutionary point and the type of speech act. It should be evident that the point of the speech act is just another name for the speech act type and does not add any more distinguishing information for the individuation of that type from the others. Fence, one can conlude that it is the information in the remaining three dimensions ( the direction of fit, pyschological state and propositional content) that really distinguish the speech act types considered by Searle.

One is still left with the nagging question: But, what is the force of an utterance? Well, if we have an utterance $u$ $=\Gamma(p)$, and, if $u$ is a directive, then $u=! \uparrow W(H$ does A), where the force $\mathrm{F}=! \uparrow \mathrm{W}$ and $\mathrm{p}=H$ does $\mathrm{A}$. But, if we are right about the irrelevance of the illocutionary point ! to the classification, then the force of a directive becones $F=\uparrow W$ and the utterance $u$ becomes $u=$ $F(p)=\uparrow W(H \operatorname{does} A)$.

However, one can plausibly argue that the sincerity condition $W$, that the speaker want the action indicated by the propositional content, is really not part of the force of the utterance. For the utterance is understood by the hearer whether the speaker wants the action or not. For example, it may well be that a general is forced to order his troops to war, after a declaration of war by the president. The general's command still has its meaning and effect irrespective of his personal wants. And, indeed, a little reflection should convince the reader that the want $W$ does not contribute to the differentiation of the typology. So now we have reduced the force of a directive to a single parameter value the direction of fit $\uparrow$.

If we are so impertinent as to ask What is the direction of fit?, we will find no ready answer that is not circular. The direction of fit of a directive is "world-towords" $\uparrow$. In other words, the force of a directive is to change the world using words. Or, better, to influence an agent to change the world by communicating a directive to that agent. In effect, in spite of all the symbols, the force remains essentially an unanalyzed notion. Little more is said than that the force of a directive is differenct from the force of an assertion. Nothing new there. Directives are used to change the world. Assertions are used to describe the world. True, but it is not enlightening. Hence, we have shown some of the dimensions to be redundant, vague and of questionable computational usefulness. We have also shown that the attempt to reduce the point feature to the remaining features leads to a dead end.

At the heart of these problems lies a more devastating pathology: We have seen that the point of the speech act is redundant for Searle's classification of speech acts. However, the point of the speech act is that part of the force that gives the central purpose of the act. Searle and Vanderveken state that the point of a speech act is a clear enough concept to be used as a primitive notion in the formal theory. "The notion of illocutionary point is the fundamental undefined primitive notion of illocutionary logic." [p.37, Searle and Vanderveken 85] Yet a few lines later they say the analysis of the point of speech acts requires a theory of mental states, specifically, a theory of intentionality which they say is beyond the scope of the book. Thus, the point of a speech act is a very complicated notion that requires a formal theory of psychology before that notion can be made formally rigorous. They also state that the point of a speech act is the most important component of the force [p.14, Searle and Vanderveken 85]. Since the force of a speech act is the most important element that differentiates speech act theory from traditional referential semantics, the point of the speech act is the central theoretical notion in the foundations of the theory of speech acts. Yet it is the point of the speech act that is left unanalyzed in Searle's formal account.

I'o sum up, the features constituting the notion of force are not motivated by any coherent theory of the meaning of speech acts. As Searle and Vanderveken admit, they have no semantics for the two most central features in the definition of force, namely, the point and direction of fit of the speech act. Instead, they leave these notions primitive and unanalyzed. That, however, amounts to leaving the notion of force an unanalyzed concept. As Searle himself states, a proper theory of force requires a theory of intention. We outline such a theory of intention in this paper and use it to formally define the force of a speech act.

745 


\section{Speech Acts in Communication}

The necessity of social cooperation gives birth to communication. The reason that utterances have the effect of actions is because they influence the cognitive state of the conversants. It is the harmony of the cognitive states of agents that makes possible cooperative social action and forms the basis of society [Werner $88 \mathrm{a}, \mathrm{b}$ ].

On our view the meaning of the speech act is best understood if we understand how the speech act is meant to influence the cognitive states of the conversants in the context of a social activity. The force of a speech act lies in its unique distribution of effect on the cognitive substates of the social agents.

One objection to our view may be that the theory of how a speech act effects the hearer is the study of perlocutionary effect. The perlocutionary effect is subject to the idiosyncrasies of individual performance and understanding and, therefore, cannot be the meaning of the speech act. We think differently. One must make a distinction, analoguous to Chomky's for syntax, between the ideal competence of the understanding subject (i.e., the ability of the subject to understand the speech act) and the actual cognitive performance. The meaning of a speech act is described by how it is to effect the ideal cognitive state of the conversants, given that the message is accepted. (see Perrault [87] for a similar view)

\section{Critique of Habermas}

Habermas [81] suggests that to get a theory of meaning for all sentences of a natural language, we have to generalize truth conditions to general satisfaction conditions. 'This would imply that assertions are given truth conditions, moral assertions being right or wrong are given rightness conditions and intention statements being sincere or not are given sincerity conditions. Since comands are legitimate or not they would, presumably, be given legitimacy conditions.

Habermas' hypothesis is based on a confusion between presuppositions (rightness, sincerity, legitimacy), truth conditions, and a theory of meaning. The general problem is that the rightness, sincerity and legitimacy conditions are not sufficient to specify and differentiate the meaning of one sentence from another. The general satisfaction conditions are inadequate for meeting the requirements of a semantic theory. Consider the example, I will go to the market tomorrow. Here the intention expressed may or may not be sincere. Even if it is sincere, this sincerity in itself does not distinguish it from $I \mathrm{am}$ going to go fly a kite. The content and point of the sentence is missed. The sincerety condition is independent of the meaning of the sentence. It says nothing of the semantic theory that specifies the semantic content and pragmantic point.

I will VP, where VP is some verb phrase describing some action, is sincere if the speaker's intentions $S_{A}$ are modified to be in accord with the meaning of the sentence. You should not have done $X$ is right if the act $\mathrm{X}$ is not allowed by the prevailing norms. Here again the rightness by itself is not enough to determine the meaning of the normative claim. The comand Attack! is legitimate or not depending on if the requisite roles and authority relations exist and are accepted by all parties concerned.
But this legitimation condition does not distinguish Attack! from Retreat! nor from any other comand. The legitimation claim that is raised and presupposed by giving a comand does not specify the meaning of the comand itself.

There is a false analogy drawn between the general satisfaction conditions and truth conditions. While truth conditions do differentiate assertive sentences that have distinct meanings, a truth conditional semantics does not give the meaning of a sentence explicitly. They do not construct a semantical object that is meant to be the sentence's meaning. Classical non-situational semantics of basic sentences did not differentiate the meanings of sentences except indirectly through differences in the content and form of the truth conditions. However, in situation semantics, where basic sentences refer to situations, we can refer to and differentiate sentence meanings directly. And that is point! The meaning of the basic sentence is already given by the deeper situation semantics. The truth conditions are defined by presupposing the deep meaning of the sentence is already given. So too for all the generalized satisfaction conditions. The deep semantics and the pragmatic interpretation of the sentence in question is presupposed in the specification/defintion of the satisfaction conditions.

A crucially important conclusion follows from the above argument: It is that conditions of use of a sentence in the sense of Wittgenstein, Austin, Searle, Cohen and Perrault and Habermas, are only indirectly related to the meaning of an utterance. Meaning is not identical with the conditions of use of the sentence. It is not identical to the preconditions of the speech action. Instead, the conditions of use of a sentence presuppose the situational and pragmatic meaning of the sentence.

\section{Psychology and Force}

Bring me a brick! and You brought me a brick may both refer to the same action. While their semantic reference is the same, their force is clearly distinct. The example shows that the meaning of a sentence cannot be identified with its reference. For in the example both sentences have the same reference, namely, the action of bringing the brick. The difference in meaning lies in the difference in the force, and, as we will see below, in the difference in their pragmatic interpretation.

To explain this difference in force we will give a pragmatic interpretation to the sentence. A comand steers action by affecting the intentions of the hearer. Its point is to affect those intentions. The assertion, on the other hand, affects the information state of the hearer and its point is to give information. This difference of force is not explainable by a purely referential theory. It requires explicit or implicit reference to mental states. Thus, if we take situation semantics to be the claim that the meaning of a sentence is the external situation it refers to, then situation semantics can give no explanation of force. If it tries to, it must bring in mental states. Thereby, it is no longer a purely referential theory. However, as we will see situation semantics serves as a foundation for understanding and defining the propositional content of speech acts. 
A proper theory of speech act force requires a formal titeory of psychology. Such a theory of psychology must be formal because its variables, mental states, will be used in the formal analysis of the point. Furthermore, a formal vsychology should show the theoretical relationships between the mental states. Thus these mental states must have a suificient complexity to account for these interrelations and to explain the linguistic phenomena. Such a theory should provide the conceptual foundations needed for the central and most important concept in ayeech act heory. In addition, it must account for the relationship between linguistic communication and social cooperation.

'To get : theory of meaning for speech acts we thus will need a theory of the intentional, informational, evaluative, and more generally, the cognitive states of the conversants. For a more detailed account of the general theory of conmunication see Werner[88a].

\section{Cognitive States}

Let 'W' be the set of time periods $t$ where time instants are ordered by a relation $<$. Let Hist $(\Omega)$ be the set of possible partial histories $\mathrm{HIt}^{\mathrm{t}}$ up to time $\mathrm{t}$. Let $\Omega$ be the set of all complete histories or worlds $H$. Worlds are series of conplete situations. (See Barwise and Perry [83].) A situation $\mathrm{s}$ is realized in $\mathrm{H}$ at $\mathrm{t}$ if $\mathrm{s}$ is contained in $\mathrm{H}$ and the domaine of $s$ is time period $t$. STRAT is the set of all possible strategies. $\mathrm{n}^{*}$ is the set of possible histories consistent with the strategy $n$. The cognitive or pepreseniational state $R$ of a conversational participant cp is described by three components $\mathrm{R}=<\mathrm{I}$, $S, V>, J$ is the information state of the $\mathrm{cp} . I$ is formalized as a set of possible partial histories. $S$ is the intentional state of the $\mathrm{cp} . \mathrm{S}$ is a set of possible strategies that guide the actions of the $\mathrm{cp}$. $V$ is the evaluative state of the cp. $V$ represents the cp's evaluation and focus on situations. The representational state $R_{A}$ may include the agent $A^{\prime} s$ representation of $B$ 's representation, $R_{A}^{B}$. It may also include the agent $A^{\prime} s$ representation of $B^{\prime} s$ representation of $A^{\prime} s$ representation, $R^{B A}{ }_{A}$. Thus we can represent arbitrary levels of nesting of representations. Let INF, INT, and EVAL be the set of all possible information, intentional, and evaluative states, respectively. Let the representational capacity, Rep, be the set of all possible representational states of a cp. For the theoretical foundations and some of the principles intorrelating intention, information and ability see Werner [88e].

\section{Representational Semantics}

A theory of the meaning of specch acts is a theory of how the representations of the cp are updated by the communicutive process. The propositional content of the speech act is given by a situation semantics in the style of Marwise and Perry [83]. We call the theory of how representational or cognitive states are transformed by rnessages the pragmatics or representational semsntics. Given a language $L$ we define a pragmatic operator Prag, where for each sentence $\alpha$ in $L$, Prag(a) is a function from Rep into Rep. Thus Prag takes a given subrepresentational state such as $I$ in $R$ and transforms it into a new substate I' $=$ Prag( $(\alpha)(I)$.

\section{Communication}

\subsection{Syntax}

To illustrate how the representational semantics works we develop the pragmatic interpretation for a small temporal propositional language fragment we will call $\mathrm{L}_{p_{t}}$. The language $I_{b_{t}}$ will include logical and temporal connectives: $\wedge$ (=and), $\vee(=$ or $), \neg$ ( $=$ not), $\wedge \Rightarrow(=$ and then), while $(=$ while). From atomic formulas $p, q$, we build up complex formulas $\alpha \wedge \beta, \alpha \vee \beta, \neg \alpha, \alpha \wedge \beta$, and $\alpha$ while $\beta$ in the usual way.

\subsection{Pragmatic Interpretation of Assertions}

For any formula $\alpha$ in $L, \operatorname{Prag}(\alpha):$ Rep $\Rightarrow$ Rep is a function that distributes over the representational state $R \in$ Rep subject to the constraints that for all $I \in I N F, S$ $\epsilon$ INT , and $V \in V A L$, then $\operatorname{Prag}(\alpha)(I) \in \mathrm{INF}$, Prag( $(\alpha)(S)$ $\in \mathrm{IN} T$, and $\operatorname{Prag}(\alpha)(\mathrm{V}) \in \mathrm{VAL}$, respectively. Below we assume Holds $(a, H, t)$ is defined by induction in the usual way where for atomic formulas Holds $(\alpha, H, t)$ if the situation referred to by $\alpha$ is realized in $\mathrm{H}$ at $\mathrm{t}$.

Prag must additionally satisfy other conditions : For atomic formulas

$\operatorname{Prag}(\alpha): \operatorname{Rep} \Rightarrow$ Rep . Let Prag be defined for the formulas $\alpha$ and $\beta$.

Prag acts on information states as follows:

$$
\begin{aligned}
& \operatorname{Prag}(\alpha \wedge \beta)(I)=\operatorname{Prag}(\alpha)(I) \cap \operatorname{Prag}(\beta)(I) \\
& \operatorname{Prag}(\alpha \vee \beta)(I)=\operatorname{Prag}(\alpha)(I) \cup \operatorname{Prag}(\beta)(I) \\
& \operatorname{Prag}(\neg \alpha)(I)=I-\operatorname{Prag}(\alpha)(I)
\end{aligned}
$$

Prag $(\alpha \wedge \Rightarrow \beta)(\mathrm{I})=\left\{\mathrm{H}^{\mathrm{t}}: \mathrm{Ht}_{\mathrm{t}} \in \mathrm{I}\right.$ and there exist times $t_{0}, t^{\prime} \varepsilon$ TimePeriods, TP, where Holds $(a, H$, $\left.t_{0}\right)$ and Holds $\left(\beta, H, t^{\prime}\right)$ and $\left.t_{0}<t^{\prime}\right\}$

Prag $(\alpha$ while $\beta)(\mathrm{I})=\left\{\mathrm{H}^{\mathrm{t}}: \mathrm{H}^{\mathrm{t}} \varepsilon \mathrm{I}\right.$ and for all $\mathrm{t}_{0}$, $t^{\prime} \varepsilon$ TP, if $t_{0}$ contains $t^{\prime}$ then if Holds $\left(\beta, H, t^{\prime}\right)$ then Holds $\left.\left(a, H, t_{0}\right)\right\}$

For example, the pragmatic interpretation of the sentence $\alpha=$ 'Jon opened the door' is arrived at as follows: a refers to the event of Jon opening the door. Prag(a) is an operator on the hearer's information state I such that Prag( $\alpha) I$ is the reduction of the set I to those histories where the event referred to by $\alpha$ occurred. The hearer $A$ knows $\alpha$ if $\alpha$ holds in all the worlds in I. Thus, A comes to know that $\alpha$ as a result of receiving and interpreting the message $a$.

\subsection{Pragmatic Interpretation of Directives}

Prag acts on the intentional substates as follows:

$$
\begin{aligned}
& \operatorname{Prag}(\alpha \wedge \beta)(S)=\operatorname{Prag}(\alpha)(S) \cap \operatorname{Prag}(\beta)(S) \\
& \operatorname{Prag}(\alpha \vee \beta)(S)=\operatorname{Prag}(\alpha)(S) \cup \operatorname{Prag}(\beta)(S) \\
& \operatorname{Prag}(\neg \alpha)(S)=S-\operatorname{Prag}(\alpha)(S)
\end{aligned}
$$$$
\text { Prag }(a \wedge \Rightarrow \beta)(S)=\left\{n \text { : for all } H \varepsilon \pi^{*}\right. \text { and }
$$
there exist times $t_{0}, t^{\prime} \varepsilon$ TP where Holds $\left(\alpha, H, t_{0}\right)$ and Holds $\left(\beta, H, t^{\prime}\right)$ and $\left.t_{0}<t^{\prime}\right\}$

Prag ( $\alpha$ while $\beta)(S)=\left(n\right.$ : for all $H \in \mathrm{n}^{*}$, exists $t, t^{\prime}$ E TP such that Holds $(a, H, t)$ and Holds $(a, H$, $\left.t^{\prime \prime}\right)$ and $t^{\prime}$ contains $t$. 
For example, if $a:=$ 'Open the door', a refers to the situation of the addressee $A$ opening the door. Prag(a) operates on $A$ 's intentional state $S_{A}$ such that $A$ opens the door. Prag does this by removing all those possible plans of $A$ that do not force $a$. Viewed constructively, a plan is incrementally built up by the Prag algorithm. And those are the plans $n$ that have some world $H \varepsilon \Pi^{*}$ where the situation referred to by $\alpha$ is not realized in $\mathrm{H}$. The result is that the agent performs the directive in parallel to other goals he may have. Again, we are talking about the ideal pragmatic competence.

Note that Prag describes the pragmatic competence of an ideal speaker and not the actual performance. He may for various reasons not accept the message. But for him to understand the conventional meaning of the assertion or directive, the conversational participant must know what the effect of the message is supposed to be if he were to accept it. Thus, a participant will not just have an actual informational and intentional state $I$ and $S$ but also hypothetical representational states HI and HS that are used to compute the pragmatic effect of a given message. If the participant then accepts the message, HI or HS will become a part of the actual representational state $R=(I, S, V)$.

\section{Pragmatic Operators}

The interpretation of utterances is actually more complicated. For a given formula a are associated several operators that give different kinds of information. Let $f_{a}$ $=\operatorname{Prag}(\alpha)$ the interpretation of $\alpha$. To describe the act - of saying a we introduce another operator. acta is the operator giving the infomation that a was just said. $\tau_{a}$ is the time operator that shifts time according to how long it took to say $a$. Here we assume for simplicity that the act of uttering a takes one unit of time. We could of course relativize the time operator to $a$. Note, $\tau_{a} \tau_{a} \neq \tau_{a}$. Combining operators, act $\boldsymbol{a}_{\alpha}$ is then the act of uttering $\alpha$ - Note, acta $\tau_{a} \neq \tau_{a} a c t_{a} . f_{a} a c t_{a} \tau_{a}$ is the act of uttering and interpreting $a$.

We have several cases possible when $A$ asserts the informative $a$ to the audience $B$.

1. If $B$ hears, understands and accepts $a$ then $I_{B} \Rightarrow f_{a} a c t_{a} \tau_{a} I_{B}$.

2. If $B$ hears, but does not understand or accept $a$, then $I_{B} \Rightarrow \operatorname{act}_{\alpha} \tau_{a} I_{B}$.

Note, if $\alpha$ is a directive then $f_{a}$ acts on $S_{B}$ and not on $I_{B}$. Yet it is known what was said. So here too,

$$
\mathrm{I}_{\mathrm{B}} \Rightarrow \operatorname{act}_{\mathbf{a}} \tau_{\mathbf{a}} \mathrm{I}_{\mathrm{B}} \text {. }
$$

3. If $B$ hears something was said, but does not know what was said, then $I_{B} \Rightarrow \tau_{\alpha} I_{B}$.

4. More odd is the case where $B$ does not hear a but gets the information of what was said subliminally and knows time passed. Then $I_{B} \Rightarrow f_{a} \tau_{\alpha} I_{B}$

The full interpretation of a for the audience $B$ depends on its force. Let $R_{B}=\left(I_{B}, S_{B}, V_{B}\right)$. Given that the message is accepted, some the cases are as follows:

1. Assertions: $\left(\mathrm{I}_{B}, \mathrm{~S}_{\mathrm{B}}, \mathrm{V}_{\mathrm{B}}\right) \Rightarrow\left(\mathrm{f}_{a} \mathrm{act}_{\mathrm{a}} \tau_{a} \mathrm{I}_{\mathrm{B}}, \mathrm{S}_{\mathrm{B}}, \mathrm{V}_{\mathrm{B}}\right)$

2. Comands: $\left(I_{B}, S_{B}, V_{B}\right) \Rightarrow\left(\operatorname{act}_{a} \tau_{a} I_{B}, f_{a} S_{B}, V_{B}\right)$
3. Statements of Intention:

$$
\left(I_{B}, S_{B}, V_{B}\right) \Rightarrow\left(\operatorname{act}_{a} \tau_{\alpha} I_{B}, f_{\alpha} S_{B}{ }^{A}, V_{B}\right)
$$

4. Statements of Value:

$$
\left(I_{B}, S_{B}, V_{B}\right) \Rightarrow\left(a_{a c t} v_{a} I_{B}, S_{B}, f_{a} V_{B}{ }^{A}\right)
$$

Below we will simplify matters by usually suppressing the action operator acto as well as the time operator $\tau_{a}$ where the above cases indicate how the a more complete description would look like. Note too, that the discourse situation $d$ can be viewed as yet another operator acting on the information state $I_{C P}$ of the coversational participants $c p=A, B$. Since there are points of view, it is clear that the operator $d$ is also a function of the conversational participant and more generally of the role of the $\mathrm{cp}$. Similarly, as we will see, the pargmatic operator is also a function of the role of the $\mathrm{cp}$ in question.

\section{Speech Act Theory}

We now give a semantic, pragmatic description of some of the speech acts in Searle's taxonomy. First some needed definitions. Given our formal pragmatics Prag for an utterance $\langle s, \alpha, h\rangle$, where $s$ is the speaker, $a$ is the sentence expressed and $h$ is the hearer in the discourse situation $d$, let the speaker $s$ have representational state $R_{\mathrm{s}}=\left(\mathrm{I}_{\mathrm{s}}, \mathrm{S}_{\mathrm{s}}, \mathrm{V}_{\mathrm{s}}\right)$ and the hearer $\mathrm{h}$ have representational state $R_{h}=\left(I_{h}, S_{h}, V_{h}\right)$. The different kinds of speech acts can be differentiated by how they effect the cognitive state of the conversants. Specifically, the force of a speech act is the set of subrepresentations in $\mathrm{R}$ that are to be transformed by the speech act. An intentional state $S$ forces a goal $\mathrm{g}$, in symbols, $S \| \Rightarrow$ iff for all $n \varepsilon S$, $n$ forces $g$, i.e., iff for all $\mathrm{H} \varepsilon \mathrm{n}^{*}, \mathrm{~g}$ is realized in $\mathrm{H}$. By definition the composite $\mathrm{S}+$ $S^{\prime}$ of two intentional states $S, S^{\prime}$ together force a goal $g$, in symbols, $S+S^{\prime} \| \Rightarrow g$ iff for all n $\varepsilon S$, no $\varepsilon S^{\prime}$, and for all $H \varepsilon n^{*} \cap n^{*}, g$ is realized in $H$. Below we will use the shorthand notation of aI for Prag(a)I $=f_{G I}$.

1. Assertives: Example: Bill opened the door.

$$
\text { 1.1. } \mathrm{I}_{\mathrm{h} . \mathrm{a}} \Rightarrow a \mathrm{I}_{\mathrm{h}} \quad \text { 1.2. } \mathrm{I}_{\mathrm{s}} \mathrm{h}_{a} \Rightarrow \alpha \mathrm{I}_{\mathrm{s}} \mathrm{h}
$$$$
\text { 1.3. } \mathrm{I}_{\mathrm{S}} \| a
$$

Remark: $I_{h}$ transforms to $\alpha_{h}$. Assertives effect the informational state of the hearer. They also effect the hearer's representation of the speaker's beliefs. The sincerity condition that the speaker believe what he asserts is expressed by $I_{s} \| \Rightarrow$.

\section{Directives: Example: Open the door!}

$$
\begin{aligned}
& \text { 2.1. } \mathrm{S}_{\mathrm{h} a} \Rightarrow \mathrm{aS}_{\mathrm{h}} \quad \text { 2.2. } \quad \mathrm{S}_{\mathrm{s}}{ }_{\mathrm{a}} \Rightarrow \mathrm{aS}_{\mathrm{s}}{ }^{\mathrm{h}} \\
& \text { 2.3 } \mathrm{I}_{\mathrm{CP}} \Rightarrow \text { act }_{\mathrm{a}} \mathrm{I}_{\mathrm{a}} \mathrm{I}_{\mathrm{CP}}
\end{aligned}
$$

Remark: The comand updates the hearer's intentions to $a S_{h}$ where $h$ does the action $a$. $a S_{s}{ }^{h}$ describes the speaker's representation of the hearer's new intentions.

3. Commissives: Example: I will open the door.
3.1. $\quad \mathrm{S}_{\mathrm{s} a} \Rightarrow \mathrm{aS}_{\mathrm{s}}$
3.2. $\mathrm{S}_{\mathrm{h}^{\mathrm{B}}} \Rightarrow a \mathrm{~S}_{\mathrm{h}^{\mathrm{s}}}$

Remark: The speaker commits himself to following those strategies that insure the propositional content of a ,i.e., all the worlds in each $\mathrm{n}^{*}$ realize the action referred to by $\alpha$. $\alpha S_{h}{ }^{8}$ represents the hearer's resulting representation of the speaker's modified intentions. 


\section{Declarations:}

Cixample: I resign, Your fired.
4.1. $I_{h} \Rightarrow a I_{h}$
4.2. $\mathrm{I}_{\mathrm{g}} a \Rightarrow a \mathrm{I}_{\mathrm{s}}$
4.3. $S_{\mathrm{S} a} \Rightarrow a S_{\mathrm{S}} 4.4$. $\mathrm{S}_{\mathrm{h}} \mathrm{a} \Rightarrow a \mathrm{~S}_{\mathrm{h}}$
4.5. $S_{\text {institution } a} \Rightarrow a S_{\text {institution }}$

Remark: 'The both hearer and speaker update their information states to $\alpha I_{h}$ and $a I_{s}$, respectively, where they know the resulting state brought on by the declaration. Furthermore, a declaration such as "you're fired" has specific intentional consequences such as no longer being paid. $a S_{\text {in:titution indicates that the declaration also has }}$ institutionul effects. Namely, it effects the composite intentions of all those with roles involved in the exployment relationship. As we mentioned above, the Prag operator is also a function of the role the $\mathrm{cp}$ has. Viewed froin another perspective, the same speech act has different effects on the intentions of different cp's according to the roles they occupy.

\section{Itepresentative Declaratives:}

Example: I find you guilty.

$$
\begin{aligned}
& \text { 5.1. } \mathrm{I}_{\mathrm{h} a} \Rightarrow a \mathrm{I}_{\mathrm{h}} \quad \text { 5.2. } \quad \mathrm{I}_{\mathrm{S} a} \Rightarrow a \mathrm{I}_{\mathrm{S}} \\
& \text { 5.3. } \mathrm{S}_{\mathrm{s} \alpha} \Rightarrow \alpha \mathrm{S}_{\mathrm{S}} \quad \text { 5.4. } \quad \mathrm{S}_{\mathrm{h}} \Rightarrow \alpha \mathrm{S}_{\mathrm{h}} \\
& \text { 5.5. } \mathbf{S} \text { institution } \alpha \Rightarrow \alpha \mathbf{S}_{\text {institution }} \\
& \text { 5.6. } \quad I_{s} \| a
\end{aligned}
$$

Remark: The representative declarative differs from the declaration in that the former must be based on certain facts obtaining. $I_{\mathrm{s}} \| \Rightarrow a$ expresses this condition. Again we ste how social roles in an institution are affected by a declaration. The judge's declaration of guilt and sentencing has very specific intentional consequences for the police and parole board, etc. These complex intentions are packed into the composite institutional role structure $\alpha \mathbf{S}_{\text {institution. What is so interesting is that our formalism }}$ allows us to talk about such complex social processes. It takes a small step toward a better understanding of the relationship between linguistic communication and social structure. It is this property of our theory that makes it a promising candidate for the design of the complex systems being contemplated in distributed artificial intelligence.

\section{Conclusion}

We have developed the outlines of a formal theory of meaning (semantics and pragmatics) of speech acts. To accomplish this we developed a formal theory of intentional states. We then related language to information and intention states. We found that meaning is not identical to conditions of use, and that meaning is not identical to reference. The formal theory of information and intention made it possible for us to construct a rigorous theory of the force of illocutionary acts. The illocutionary force is defined in terms of the specific subrepresentations that the speech act is to modify. 'The subrepresentations are only sketched. But the point of the approach is quite clear. The cognitive states of the conversational participants, for example, system and user, play a dominant role in the theory of meaning and force of speech acts. An actual implementution of an algorithm for Prag and an actual knowledge representation scheme to describe the information, intentional, and evaluative states requires making significantly more detailed system design decisions.

Within a wider context, we have aimed at providing a general theoretical framework for designing systems with a communicative competence using natural language. Our theory fits well with planning models in robotics. It also fits well with discourse and speech act theories. Of more global significance is the fact that our theory of communication fits well with von Neumann and Morgenstern's economic theory [see von Neumann and Morgenstern 47]. This is because our theories of communication and intention allow us to define complex social roles and social structures [Werner $88 \mathrm{a}, 88 \mathrm{~b}$ ]. This will hopefully help to clarify the complex relationship between language and society.

\section{References}

Appelt, D. E.

PLANNING ENGLISH SENTENCLS; Cambridge University Press, New York, 1985.

Barwise, J., and Perry, $J$., SITUATIONS ANI) AT'II'IUDES, Bradford Books/MI'T Press, 1983.

H. Bergmann/ M. Gerlach

Semantisch-pragmatische Verarbeitung von Äußerungen im natürlichsprachlichen Beratungssystem WISBER, in: W. Brauer, W. Wahlster (Wds.): Wissensbasierte Systeme - GIKongress 1987. Springer Verlag, Berlin 1987, pp. 318-327

Cohen, P. R, and Perrault, C. R.,

"Elements of a Plan-Baged Theory of Speech Acts," COGNITIVE SCIENCE, 3, pp. $177-212,1979$.

Grosz, B. J.,

"The Structures of Discourse Structure," Techn. Note 369 Artificial Intelligence Center, SRI International, Menlo Park, California, 1985.

Habermas, J., TIIFORIL DES KOMMUNIKATIVEN HANDISLNS, Frankfurt-a.M., 1981.

Moore, R. C.,

"Reasoning About Knowledge and Action", Tech. Note 191 Artificial Intclligence Center, SRI International, Melno Park, California, 1980.

Montague, $\mathbf{R}$.,

"The Proper Treatment of Quantification in Ordinary English", In Thomason, R., (ed.), FORMAl, PHILOSOPHY: Seleleted Papers of Richard Montague, NewHaven: Yale University Press, pp. 247-270, 1974.

Perrault, C.K.,

"An Application of Default Logic to Speech Act Theory",Report No. CSLI-87-90, CSLI, SRI International, Menlo Park, California, 1987

Searle, J. R.,

"A Taxonomy of Illocutionary Acts", in K. Gunderson (ed.) LANGUAGE, MIND, AND KNOWLEDGE, MINNESO'A STUDIES IN THE PIILOSOPHY OF SCIENCE, Vol. 7 , Minneapolis: University of Minnesota Press, 1975.

Searle, J. R., and Vanderveken, FOUNDATIONS OF ILLOCUTIONARY LOGIC Cambridge University Press, London, 1985

Von Noumann, J. and Morgenstern, $O$., THE THEORY OF GAMLS AND LCONOMIC BEHAVIOR, Princeton University P'ress, Princeton, NJ, 1947.

Werner, $\mathrm{C}$

"Toward a 'Theory of Communication and Cooperation for Multiagent Planning", THEORETICAL ASPECT'S OF REASONING ABOUT KNOWLEDGH: Procedings of the Second Conterence, Morgan Kaufman Publishers, 1988a.

Werner, $\mathrm{E}$.,

"Socializing lRobots: A Theory of Communication and Cooperation for Distributed Artificial Intelligence", to be presented at 1988 Distributed Artificial Intelligence Workshop, Lake Arrowhead, California, 19988b.

Werner, E.,

"Intention, Information and Ability: Outline of a Unified 'Theory", unpublished manuscript, 1988c. 